Research Article

\title{
Computationally Efficient Unitary ESPRIT Algorithm in Bistatic MIMO Radar
}

\author{
Baobao Liu $\left(D,{ }^{1}\right.$ Tao Xue, ${ }^{1}$ Cong $X{ }^{2},{ }^{2}$ and Yongjun Liu ${ }^{3}$ \\ ${ }^{1}$ School of Computer Science, Xi'an Polytechnic University, Xi'an 710048, China \\ ${ }^{2}$ School of Information Engineering, Xijing University, Xi'an 710123, China \\ ${ }^{3}$ School of Electronic Engineering, Xidian University, Xi'an 710071, China \\ Correspondence should be addressed to Baobao Liu; liubaobao1222@163.com
}

Received 2 March 2021; Revised 12 April 2021; Accepted 6 June 2021; Published 17 June 2021

Academic Editor: Junpeng Shi

Copyright ( $\odot 2021$ Baobao Liu et al. This is an open access article distributed under the Creative Commons Attribution License, which permits unrestricted use, distribution, and reproduction in any medium, provided the original work is properly cited.

\begin{abstract}
A low complexity unitary estimating signal parameter via rotational invariance techniques (ESPRIT) algorithm is presented for angle estimation in bistatic multiple-input-multiple-output (MIMO) radar. The devised algorithm only requires calculating two submatrices covariance matrix, which reduces the computation cost in comparison with subspace methods. Moreover, the signal subspace can be efficiently acquired by exploiting the NystrÖm method, which only needs $O\left(M N K^{2}\right)$ flops. Thus, the presented algorithm has an essentially diminished computational effort, especially useful when $K \ll M N$, while it can achieve efficient angle estimation accuracy as well as the existing algorithms. Several theoretical analysis and simulation results are provided to demonstrate the usefulness of the proposed scheme.
\end{abstract}

\section{Introduction}

Target estimation has been a significant problem in radar systems, which has been applied in widespread in sonar, guidance systems, speech processing, communication, medical signal processing, and other fields [1-3]. In recent years, considerable research interests have been drawn to MIMO radar [4-13], which exploits multiple antennas to emit diverse waveforms and utilizes multiple antennas to receive the echo signals [14]. This leads to its more underlying benefits over phased-array radar [15-17] (e.g., enhancing the spatial resolution, fading effect overcoming, and enhancing the parameter identifiability). MIMO radar can be regarded as an expansion of the phased-array radar, where the exploited waveforms are effectively independent [18]. Generally, MIMO radars can be divided into two types, the collocated MIMO radar and the statistical MIMO radar, based on the different array antenna configurations [19]. Furthermore, the collocated MIMO radars are categorised into two types, namely, the monostatic MIMO radar and the multistatic MIMO radar. Due to the fact that the emitting and receiving antennas are not in the identical location, the DOD and DOA estimation has become a considerable research matter [4, 18-21]. In our work, we mainly focus on the DOD and DOA estimation issue in the bistatic MIMO radar.

According to the recent researches, several algorithms [4-10] have been proposed for estimation angle in the bistatic MIMO radar. In [4], the reduced-dimension multiple signal classification (MUSIC) algorithm that uses onedimensional search is presented to angle estimation, which achieves high angle estimation accuracy in comparison with Capon algorithm [20]. In [5], the Capon algorithm is extended to DOD and DOA estimation, which has heavy computational complexity for requiring two-dimensional angle search. Moreover, the technique is subjected to some performance degradations for the proximate receiving steering vector. Besides, the estimation of angles needs peak searching with computational intensive. The root-MUSIC algorithm [6] without peak searching is presented by utilizing polynomial rooting technique to reduce the computational cost. In [7], the ESPRIT technique that uses the invariance technique of both the transmitting array and the receiving array is presented to estimate angle in the bistatic MIMO radar. However, the algorithm requires the pairing 
operation. In [8], to address the problem of automatic pairing, a combination ESPRIT-MUSIC algorithm is developed, which provides beneficial angle accuracy. In [9], a unitary ESRPIT technique that exploits the real-valued processing is devised for estimating angle in the bistatic MIMO radar, which has high estimation precision. In [10], the maximum likelihood algorithm is presented for direction finding estimation in MIMO radar. In [22], the novel joint angle estimation method is proposed by using tensor decomposition in the nested bistatic MIMO radar. Moreover, various methods are introduced for bistatic MIMO radar in [23-25]. However, the abovementioned algorithms have a large amount of computation since they require the calculation of sample covariance matrix (SCM) and its eigenvalue decomposition (EVD) to obtain the noise subspace or signal noise, especially for large MIMO radar array and a great deal of snapshots scenarios. In order to tackle this serious problem, a computationally efficient algorithm is devised for direction estimation in this work. Unlike the existing algorithms [4-10], the presented algorithm only requires to compute two submatrices of the SCM, which avoids calculating of SCM and its EVD by exploiting the NystrÖm technique. The proposed method can be also applied in the nonuniform linear array, L-shape array, and uniform circular array for angle estimation. The NystrÖm method has been extensively applied in speed up methods $[26,27]$ and is first utilized by Williams and Seeger [27] for sparsifying kernel matrices. By exploiting the NystrÖm method [28, 29], we extend the previous work [30] and develop a low complexity unitary ESPRIT algorithm which not only has high angle estimation precision but also obtains light computational cost, especially in large MIMO radar array scenario. In this paper, we derive a new powerful unitary ESPRIT approach, which exhibits many benefits as follows: (a) it has much lower computational cost than that of the ESRPIT and unitary ESPRIT methods; (b) it enjoys higher angle estimation precision than the ESPRIT algorithm; (c) it is suitable for direction finding estimation of large MIMO radar array. The benefits of the presented algorithm are shown by some simulation experiments.

\section{Data Model}

In this paper, we think about a bistatic MIMO radar system (Figure 1) constituted of $M$-transmitting antenna array and $N$-receiving antenna array, both of which are half-wavelength spaced uniform linear arrays [7-9]. Assume that there exist $P$ noncoherent targets located in the same range bin. The DOD and DOA of the pth target relative to the transmitting array normal and the receiving array normal are denoted by $\theta_{p}$ and $\phi_{p}(p=1,2, \ldots, P)$, respectively. Thus, the signal model can be given as $[7,8]$

$$
\mathbf{y}(t)=\mathbf{A} \mathbf{s}(t)+\mathbf{n}(t),
$$

where $\mathbf{A}=\left[\mathbf{a}_{1}, \mathbf{a}_{2}, \ldots, \mathbf{a}_{P}\right]$ denotes an $M N \times P$ matrix consisting of the $P$ steering vectors and $\mathbf{a}_{p}=\mathbf{a}_{r}\left(\phi_{p}\right) \otimes \mathbf{a}_{t}\left(\theta_{p}\right)$ illustrates the Kronecker product of the receiving array steering vector and the transmitting array steering vector for

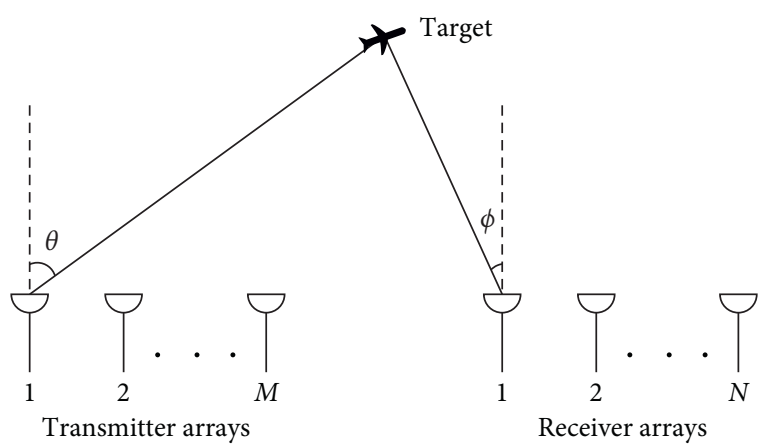

FIgURE 1: Radar configuration.

the $p$ th source. $\mathbf{a}_{r}\left(\phi_{p}\right)$ and $\mathbf{a}_{t}\left(\theta_{p}\right)$ are respectively rewritten as

$$
\begin{aligned}
& \mathbf{a}_{r}\left(\phi_{p}\right)=\left[1, \exp \left(j \pi v_{p}\right), \ldots, \exp \left(j \pi(N-1) v_{p}\right)\right]^{T}, \\
& \mathbf{a}_{t}\left(\theta_{p}\right)=\left[1, \exp \left(j \pi u_{p}\right), \ldots, \exp \left(j \pi(M-1) u_{p}\right)\right]^{T},
\end{aligned}
$$

and $u_{p}=\sin \theta_{p}, v_{p}=\sin \phi_{p}$, where $\phi_{p}$ and $\theta_{p}$ represent the DOA and DOD, respectively. $\mathbf{s}(t)=\left[s_{1}(t), s_{2}(t), \ldots, s_{P}(t)\right]^{T}$ is a column vector, in which $s_{p}(t)=\alpha_{p} e^{j 2 \pi f_{d p} t}$ denotes the envelope of the reflected signal with $\alpha_{p}$ being the amplitude containing the reflection coefficients and path losses and so on [7-9]. $\mathbf{n}(t)$ denotes an $M N \times 1$ complex Gaussian white noise vector with zero mean and covariance matrix $\sigma^{2} I_{M N}$.

\section{NystrÖm Method-Based Unitary ESPRIT for Angle Estimation}

3.1. Real-Valued Processing. In order to reduce computational complexity, we have to transform the complex data to real data by matrix method since the array, the received data, is complex data. Let $\mathbf{Y}$ be represented as the data matrix consisting of $L$ snapshots $\mathbf{y}\left(t_{l}\right), 1 \leq l \leq L$. The augmented data matrix is defined as $\mathbf{Z}=\left[\mathbf{Y}_{M N} \mathbf{Y}^{*} \Pi_{L}\right]$, where $\Pi_{M N}$ represents the exchange matrix including $J$ ones on its antidiagonal and zeros elsewhere. Then, the real-valued matrix is expressed as $[9,11]$

$$
\boldsymbol{\Gamma}=\mathbf{Q}_{M N}^{H} \mathbf{Z} \mathbf{Q}_{2 L},
$$

where $\mathbf{Q}_{J}$ signifies sparse unitary matrix, expressed as

$$
\begin{aligned}
\mathbf{Q}_{2 J} & =\frac{1}{\sqrt{2}}\left[\begin{array}{cc}
\mathbf{I}_{J} & j \mathbf{I}_{J} \\
\boldsymbol{\Pi}_{J} & -j \boldsymbol{\Pi}_{J}
\end{array}\right], \\
\mathbf{Q}_{2 J+1} & =\frac{1}{\sqrt{2}}\left[\begin{array}{ccc}
\mathbf{I}_{J} & 0 & j \mathbf{I}_{J} \\
\mathbf{0}^{T} & \sqrt{2} & \mathbf{0}^{T} \\
\boldsymbol{\Pi}_{J} & 0 & -j \boldsymbol{\Pi}_{J}
\end{array}\right] .
\end{aligned}
$$

3.2. Signal Subspace Estimation. To use the NystrÖm technique $[27,28]$ for estimating angle, we disintegrate the matrix $\Gamma$ as follows [30]: 


$$
\Gamma=\left[\begin{array}{l}
\Gamma_{1} \\
\Gamma_{2}
\end{array}\right]
$$

where $\Gamma_{1} \in \mathbb{R}^{K \times L}$ and $\Gamma_{2} \in \mathbb{R}^{(M N-K) \times L}$ are the real-valued submatrices received by the first $K$ antenna and the rest of the $(M N-K)$ antennas, respectively. We define

$$
\begin{aligned}
& \mathbf{R}_{11}=\mathbf{E}\left[\boldsymbol{\Gamma}_{1} \boldsymbol{\Gamma}_{1}^{H}\right], \\
& \mathbf{R}_{21}=\mathbf{E}\left[\boldsymbol{\Gamma}_{2} \boldsymbol{\Gamma}_{1}^{H}\right] .
\end{aligned}
$$

Moreover, we must ensure that $\mathbf{R}_{11}$ denotes full rank matrix where $K$ satisfies $\{K \mid P \leq K \leq \min (M N, L)\}$, $K=1,2, \ldots, M N$. It is noted that $K$ has not been required to ascend substantially with $M N$. For instance, when $M N$ grows from 10 to 30 , a relatively little $K$, such as $K=12$, is sufficient to insure estimating precision, which also reduces the computational complexity.

Suppose that the EVD of $\mathbf{R}_{11}$ is $\mathbf{U}_{11} \Lambda_{11} \mathbf{U}_{11}^{H}$, where $\mathbf{U}_{11} \in \mathbb{C}^{K \times K}$ denotes the eigenvector matrix and $\Lambda_{11}$ represents the diagonal matrix. Defining $\mathbf{U}_{21} \triangleq \mathbf{R}_{21} \mathbf{U}_{11} \Lambda_{11}^{-1}$, we can constitute a new matrix as follows [30]:

$$
\mathbf{U} \triangleq\left[\begin{array}{c}
\mathbf{U}_{11} \\
\mathbf{U}_{21}
\end{array}\right] \text {. }
$$

Then, according to the results from the remark, we can obtain the signal subspace without the computation of SCM and its EVD.

Remark 1 (see [30]). Suppose that the EVD of $\mathbf{G}^{H} \mathbf{G}$ is $\mathbf{U}_{G} \Lambda_{G} \mathbf{U}_{G}^{H}$ and $\mathbf{G}=\mathbf{U} \Lambda_{11}^{1 / 2}$, where $\Lambda_{G}=\operatorname{diag}\left[\lambda_{G 1}, \ldots, \lambda_{G K}\right]$ denotes the eigenvalue matrix with $\lambda_{G 1} \geq \cdots \geq \lambda_{G K}$ and $\mathbf{U}_{G}=$ $\left[\mathbf{u}_{G 1}, \ldots, \mathbf{u}_{G K}\right]$ represents the corresponding eigenvector matrix with $\mathbf{u}_{G i}(i=1, \ldots, K)$ being the $i$ th eigenvector. Then, the signal subspace is constructed by the first $P$ column vectors of $\Pi$ as follows:

$$
\operatorname{span}\left\{\mathbf{E}_{s}\right\}=\operatorname{span}\{\mathbf{A}\},
$$

where $\mathbf{E}_{s} \triangleq \Pi(:, 1: P)$ and $\Pi=\mathbf{G U}_{G}$.

3.3. Angle Estimation. Then, according to the unitary ESPRIT algorithm $[9,11]$, the real-valued invariance relation is described as follows:

$$
\mathbf{F}_{2}^{\theta} \mathbf{d}_{p}=\tan \left(\frac{\pi u_{p}}{2}\right) \mathbf{F}_{1}^{\theta} \mathbf{d}_{p}
$$

where $\mathbf{F}_{1}^{\theta}=\operatorname{Re}\left\{\mathbf{Q}_{(M-1) N}^{H} \operatorname{diag}^{N}\left\{\mathbf{J}_{2}^{\theta}\right\} \mathbf{Q}_{M N}\right\} \quad$ and $\mathbf{F}_{2}^{\theta}=\operatorname{Im}$ $\left\{\mathbf{Q}_{(M-1) N}^{H} \operatorname{diag}^{N}\left\{\mathbf{J}_{2}^{\theta}\right\} \mathbf{Q}_{M N}\right\}$ denote real-valued matrix, respectively, and $\mathbf{J}_{2}^{\theta}$ is defined in $[9,11] . \mathbf{d}_{p}=\mathbf{Q}_{M N}^{H} \mathbf{a}_{p}$ denotes a steering vector that is real-valued. Thus, $\mathbf{F}_{2}^{\theta} \mathbf{E}_{s}=\mathbf{F}_{1}^{\theta} \mathbf{E}_{s} \boldsymbol{\Psi}_{\theta}$ represents the real-valued invariance equation for the transmitter array where $\boldsymbol{\Psi}_{\theta}=\mathbf{T}^{-1} \boldsymbol{\Phi}_{\theta} \mathbf{T}$ and $\boldsymbol{\Phi}_{\theta}=\operatorname{diag}\left[\tan \left(\pi u_{1} / 2\right), \tan \left(\pi u_{1} / 2\right), \ldots, \tan \left(\pi u_{p} / 2\right)\right] \quad$ signifies a real-valued diagonal matrix whose diagonal elements include information of estimating $\operatorname{DOD}[9,11]$. In the receiving array, similarly, the real-valued invariance equation is constructed by

$$
\mathbf{F}_{2}^{\phi} \mathbf{E}_{s}=\mathbf{F}_{1}^{\phi} \mathbf{E}_{s} \Psi_{\phi}
$$

where $\mathbf{F}_{1}^{\phi}=\operatorname{Re}\left\{\mathbf{Q}_{(M-1) N}^{H} \mathbf{J}_{2}^{\phi} \mathbf{Q}_{M N}\right\} \quad$ and $\quad \mathbf{F}_{2}^{\phi}=\operatorname{Im}\left\{\mathbf{Q}_{(M-1) N}^{H}\right.$ $\left.\mathbf{J}_{2}^{\phi} \mathbf{Q}_{M N}\right\}, \quad \mathbf{J}_{1}^{\phi}=\left[\mathbf{I}_{M(N-1) \times M(N-1)} \mathbf{0}_{M(N-1) \times M}\right]$ and $\mathbf{J}_{2}^{\phi}=\left[\mathbf{0}_{M}\right.$ $\left.(N-1) \times M \mathbf{I}_{M(N-1) \times M(N-1)}\right], \Psi_{\phi}=\mathbf{T}^{-1} \Phi_{\phi} \mathbf{T}, \Phi_{\phi}=\operatorname{diag}[\tan$ $\left.\left(\pi v_{1} / 2\right), \tan \left(\pi v_{2} / 2\right), \ldots, \tan \left(\pi v_{p} / 2\right)\right]$, and $\mathbf{T}$ stands for a nonsingular matrix. $\Phi_{\theta}$ represents a real-valued diagonal matrix whose diagonal elements include information of estimating DOA. Then, $\Psi_{\theta}+j \Psi_{\varphi}$ is described as $[9,11]$

$$
\Psi_{\theta}+j \Psi_{\varphi}=\mathbf{T}^{-1}\left\{\Psi_{\theta}+j \Psi\right\} \mathbf{T} .
$$

Then, the DODs and DOAs can be estimated by

$$
\begin{aligned}
& \hat{\theta}_{p}=\arcsin \left\{2 \arctan \frac{\left(\left[\Phi_{\theta}\right]_{p p}\right)}{\pi}\right\}, \quad p=1, \ldots, P, \\
& \widehat{\phi}_{p}=\arcsin \left\{2 \arctan \frac{\left(\left[\Phi_{\phi}\right]_{p p}\right)}{\pi}\right\}, \quad p=1, \ldots, P .
\end{aligned}
$$

\section{Computational Complexity and Cramér-Rao Bound (CRB)}

The presented technique does not need utilizing the whole SCM. Instead, it requires calculating $\mathbf{R}_{11}$ and $\mathbf{R}_{21}$ which need $O\left(L K^{2}\right)$ and $O\left(M N L K-L K^{2}\right)$ flops, respectively. Meanwhile, the signal subspace is constructed by exploiting the NystrÖm approach, where the computational complexity is $O\left(M N K^{2}\right)$. Thus, the presented method requires $\mathrm{O}\left(M N L K+M N K^{2}\right)$. However, the classical unitary ESPRIT and ESPRIT algorithms need $O\left(\left(M^{2} N^{2} L+M^{3} N^{3}\right) / 4\right)$ and $O\left(M^{2} N^{2} L+M^{3} N^{3}\right)$ flops, respectively, which are much higher than $O\left(M N L K+M N K^{2}\right)$ flops on condition that $K \ll \min (M N, L)$. Furthermore, referring to [11], we use $\mathrm{CRB}$ in simulation as follows:

$$
\mathrm{CRB}=\frac{\sigma^{2}}{2 L}\left\{\operatorname{Re}\left[\mathbf{D}^{H} \boldsymbol{\Pi}_{A}^{\perp} \odot \widehat{\mathbf{P}}_{w}^{T}\right]\right\}^{-1},
$$

where 


$$
\begin{aligned}
\mathbf{D} & =\left[\frac{\partial \mathbf{a}_{1}}{\partial \theta_{1}}, \frac{\partial \mathbf{a}_{2}}{\partial \theta_{2}}, \ldots, \frac{\partial \mathbf{a}_{k}}{\partial \theta_{k}}, \frac{\partial \mathbf{a}_{1}}{\partial \phi_{1}}, \frac{\partial \mathbf{a}_{2}}{\partial \phi_{2}}, \ldots, \frac{\partial \mathbf{a}_{k}}{\partial \phi_{k}}\right], \\
\boldsymbol{\Pi}_{A}^{\perp} & =\mathbf{I}_{M N}-\mathbf{A}\left(\mathbf{A}^{H} \mathbf{A}\right)^{-1} \mathbf{A}^{H}, \\
\widehat{\mathbf{P}}_{w} & =\left[\begin{array}{ll}
\widehat{\mathbf{P}}_{s} & \widehat{\mathbf{P}}_{s} \\
\widehat{\mathbf{P}}_{s} & \widehat{\mathbf{P}}_{s}
\end{array}\right], \\
\widehat{\mathbf{P}}_{s} & =\frac{1}{L} \sum_{t=1}^{L} \mathbf{s}(t) \mathbf{s}^{H}(t) .
\end{aligned}
$$

\section{Simulation Results}

In this installment, a vast number of computer simulations are demonstrated to prove the effectiveness of the proposed technique. We compare performance of the estimating angle of the presented method with the ESPRIT algorithms [7] and unitary ESPRIT [9] and present their computational complexity analysis. In the following simulation experiments, 200 Monte-Carlo iterations are adopted for the bistatic MIMO radar in the experiments. We suppose that there exist three noncoherent targets and their location is at angles $\left(\theta_{1}, \phi_{1}\right)=\left(10^{\circ}, 20^{\circ}\right), \quad\left(\theta_{2}, \phi_{2}\right)=\left(-8^{\circ}, 30^{\circ}\right), \quad$ and $\left(\theta_{3}, \phi_{3}\right)=\left(0^{\circ}, 45^{\circ}\right)$, respectively. The root mean squared error (RMSE) of over angle [9] is exploited in the simulation experiments.

Figures 2 and 3 describe the angle estimation paired results of the presented scheme with $S N R=10 \mathrm{~dB}$ and $\mathrm{SNR}=10 \mathrm{~dB}$, respectively. It can be shown that the transmit angles (DODs) and receive angles (DOAs) can be clearly seen. Figure 3 also implies that the presented scheme can efficiently estimate angle of the targets in low SNR scenario.

Figures 4 and 5 demonstrate performance comparison of the estimating angle with $M=8, N=6$ and $M=6, N=6$, respectively. We compare the presented technique with the ESPRIT and the unitary ESPRIT methods. Figures 4 and 5 demonstrate that the proposed algorithm has much better estimation precision than the ESPRIT method and enjoys high estimation precision that is almost the same as the unitary ESPRIT scheme at high SNR range. However, the presented algorithm is somewhat inferior to the unitary ESPRIT scheme at the low SNR scenario.

Figures 6-9 show performance comparison of estimation of the presented technique with $L=50$ and $L=100$ for different $M / N$, respectively. From Figures 6-9, we can find that the angle estimation precision of the presented scheme is significantly enhanced with the number of transmitting array elements/receiving array elements increasing. Multiple receiving/transmitting array elements enhance estimation precision owing to diversity gain.

Figures 10 and 11 illustrate estimation precision comparison of the presented technique with $M=6$ and $N=6$ for different values of $L$, respectively. As shown in Figures 10 and 11, the estimation precision of the presented technique is boosted with $L$ increasing. Meanwhile, Figure 10 also indicates that the presented method has

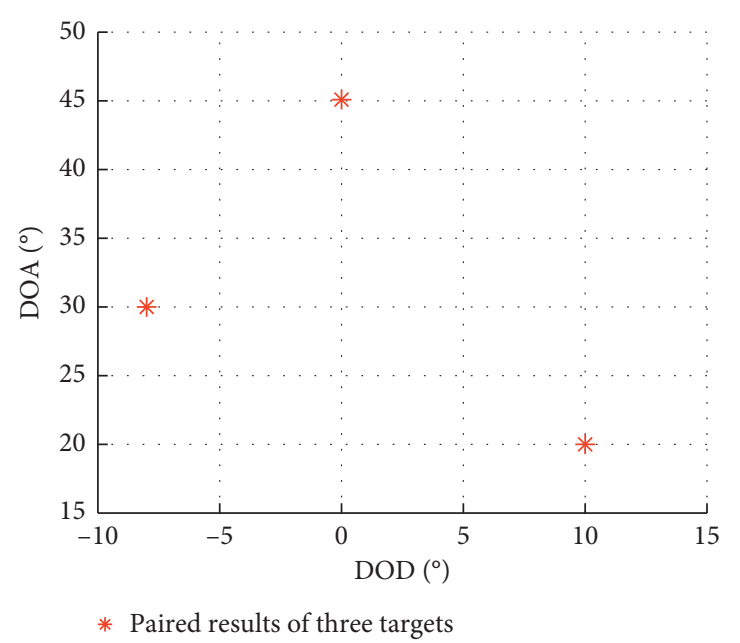

FIgURE 2: Paired results with $\mathrm{SNR}=10 \mathrm{~dB}, M=8, N=6$, and $L=200$.

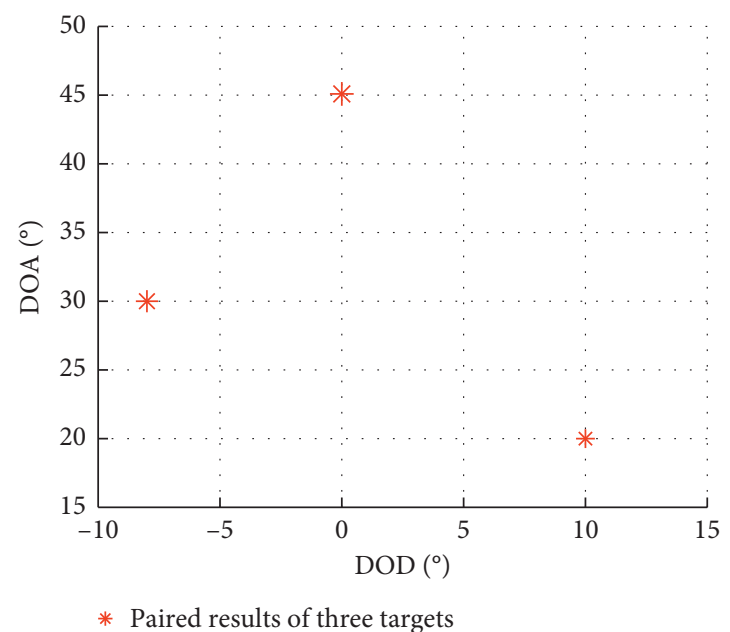

FIgURE 3: Paired results with $\mathrm{SNR}=5 \mathrm{~dB}, M=8, N=6$, and $L=200$.

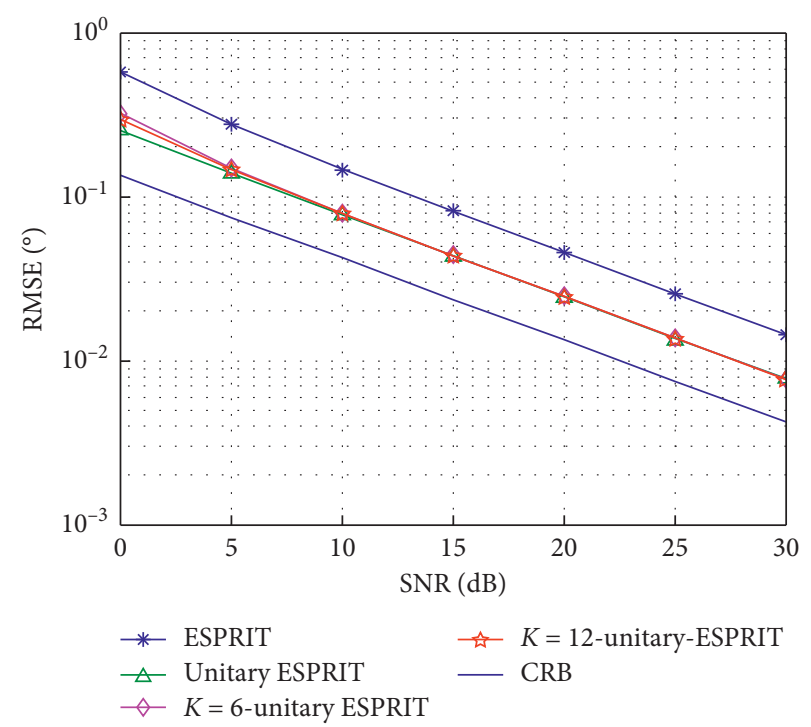

Figure 4: RMSE with $M=8, N=6$, and $L=50$. 


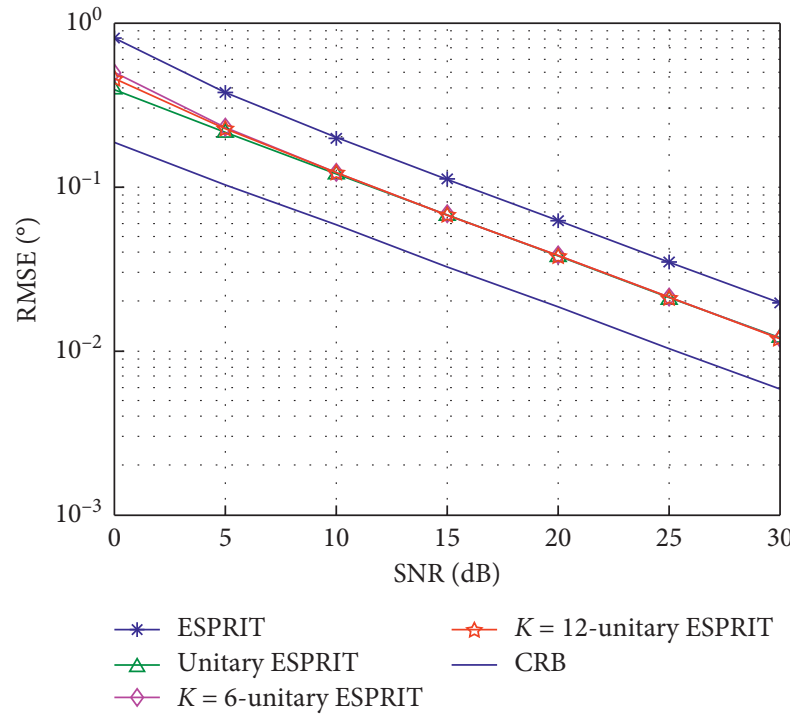

Figure 5: RMSE with $M=6, N=6$, and $L=50$.

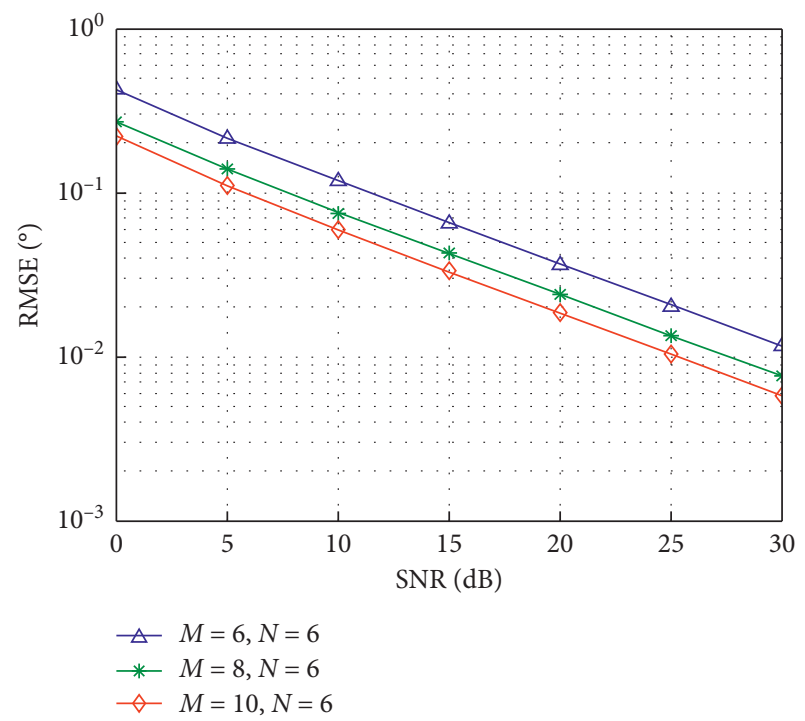

Figure 6: RMSE with $L=50$ and different $M$.

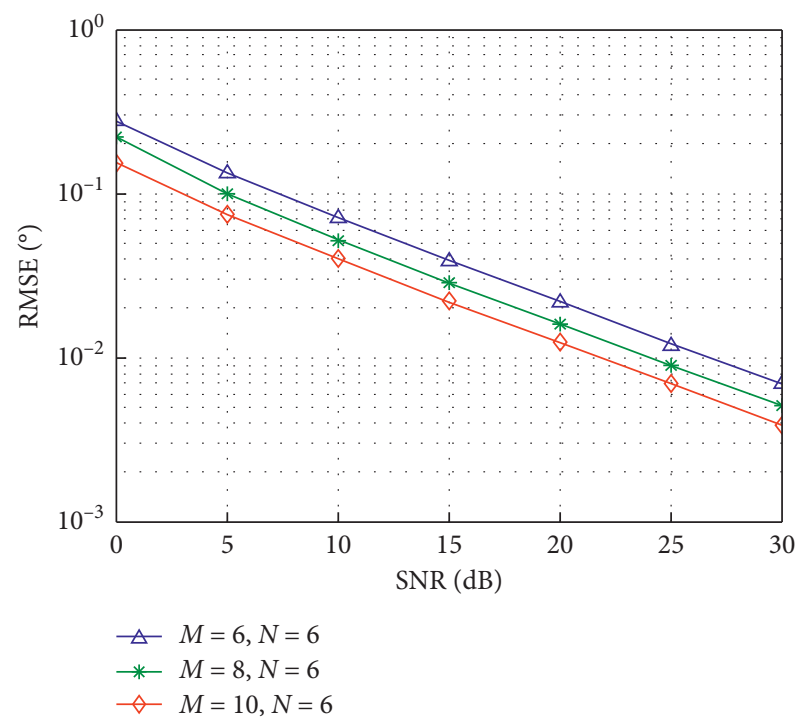

FIgURE 7: RMSE with $L=100$ and different $M$.

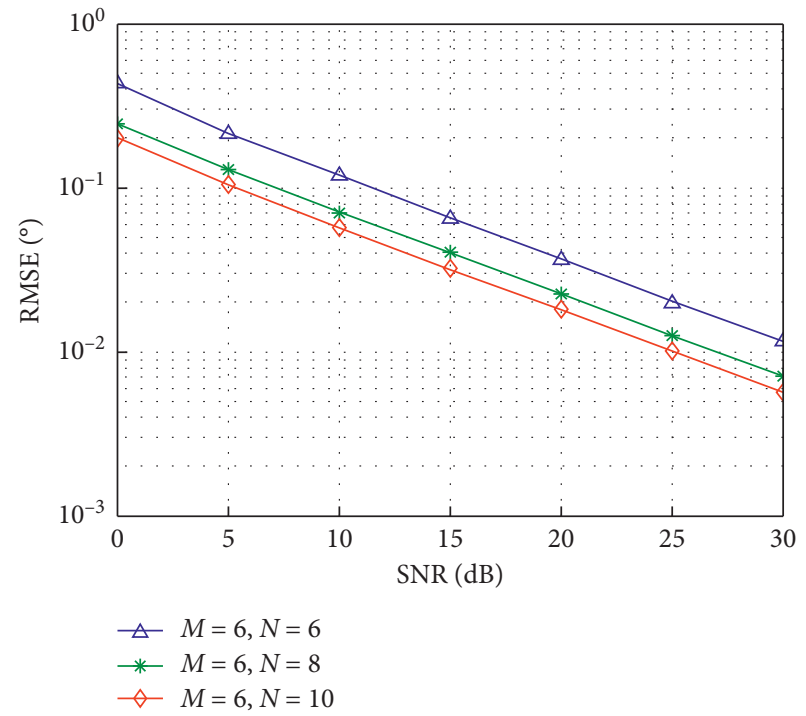

Figure 8: RMSE with $L=50$ and different $N$.

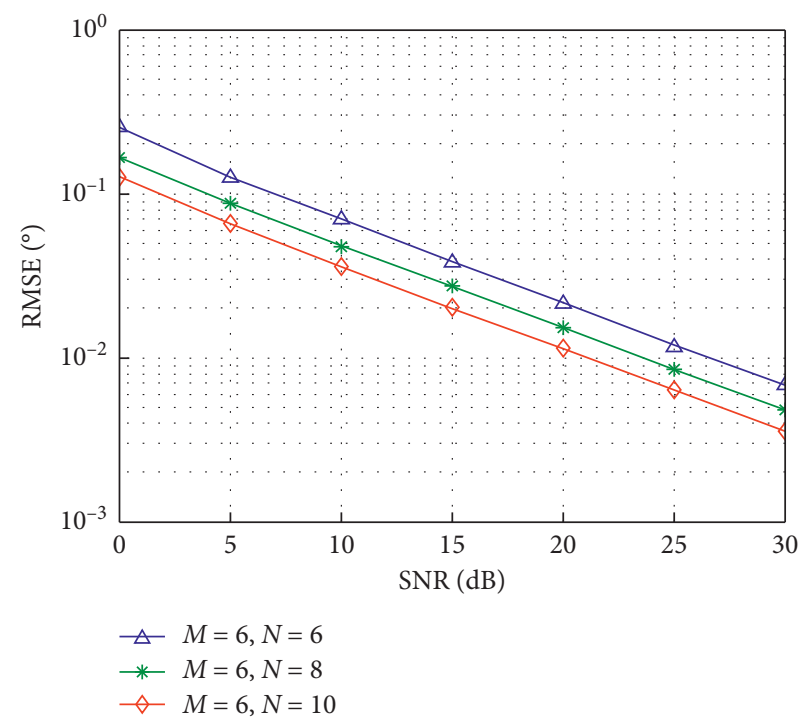

Figure 9: RMSE with $L=100$ and different $N$.

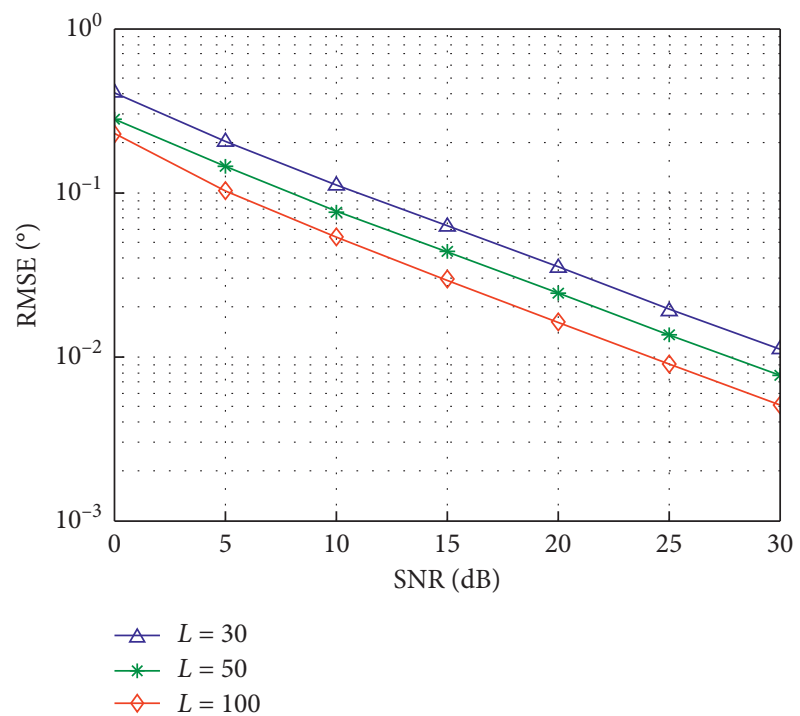

Figure 10: RMSE with $M=6, N=6$, and different $L$. 


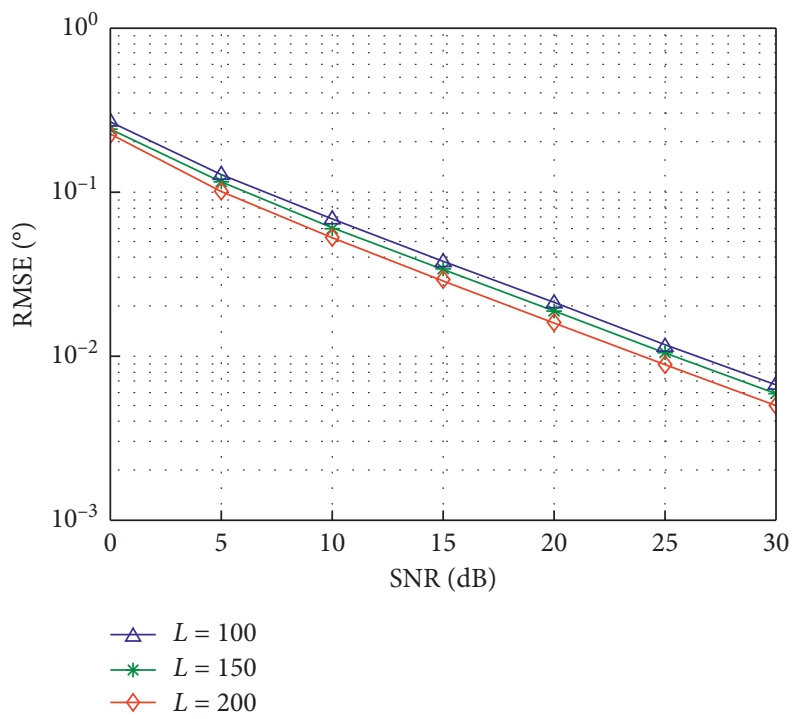

Figure 11: RMSE with $M=6, N=6$, and different $L$.

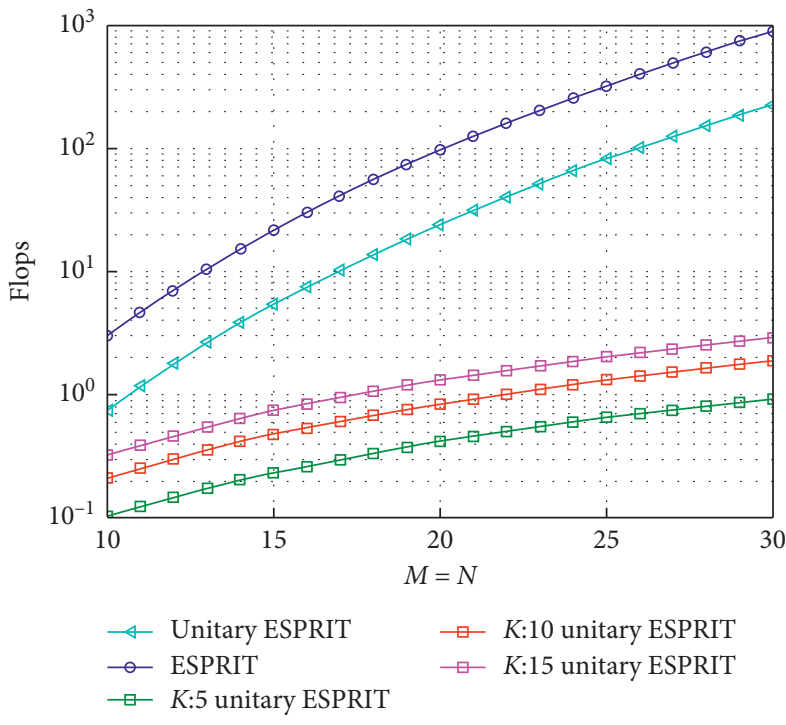

FIGURE 12: Complexity comparison with $L=200$.

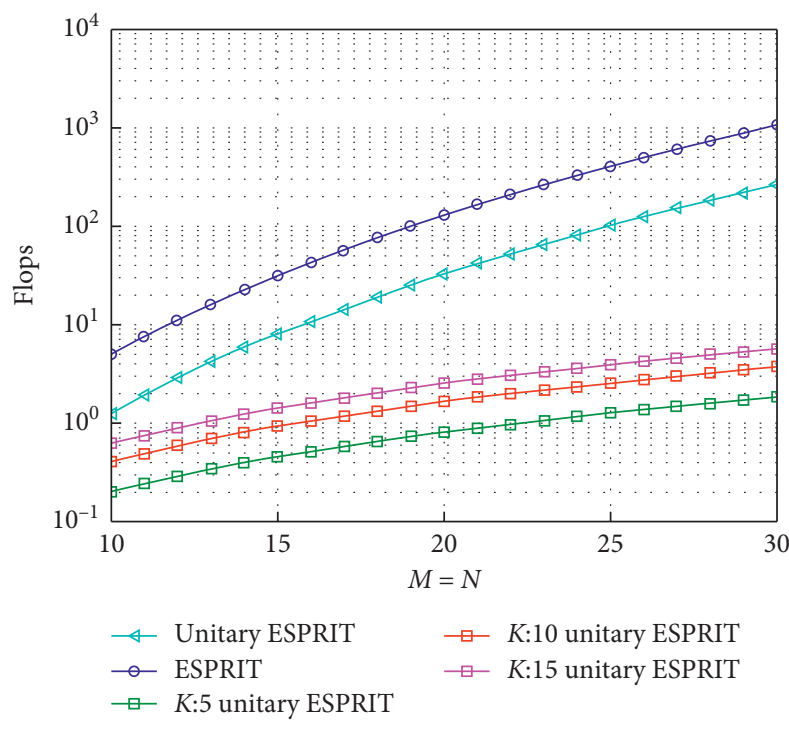

Figure 13: Complexity comparison with $L=400$. 


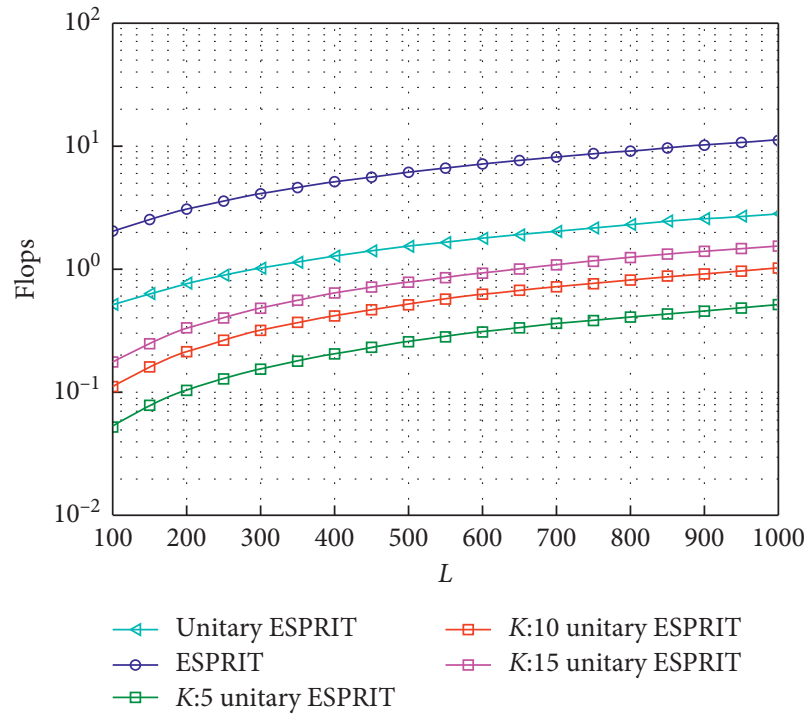

FIgURE 14: Complexity comparison against $L$ with $M=N=10$.

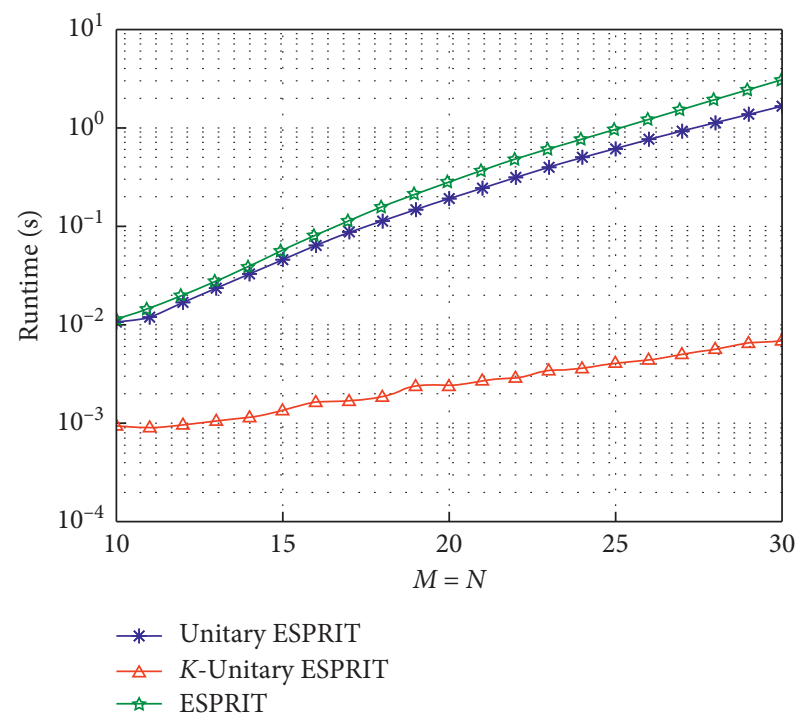

Figure 15: Runtime comparison versus $M=N$ with $L=100$ and $K=12$.

beneficial estimation precision at small number of snapshots scenario.

Figures 12-14 illustrate the complexity comparison with $K=5,10,15$, where we can find the proposed algorithm has much less computational cost in comparison with the ESPRIT and the unitary ESPRIT schemes, particularly when $M=N$ becomes larger.

Figure 15 and Table 1 describe the runtime of the three ESPRIT schemes. They depict the average CPU time required to calculate each ESPRIT approach on the personal computer with Intel(R) core(TM) 2 Duo CPU T3700 processor. We can clearly observe that the presented approach is much more computationally effective than the existing schemes, especially when $M=N$ becomes bigger.
TAble 1: Computations time comparison $(P=3, L=100, K=12$, and $\mathrm{SNR}=10 \mathrm{~dB}$ ).

\begin{tabular}{lccc}
\hline $\begin{array}{l}\text { Transmit and } \\
\text { receive elements }\end{array}$ & $\begin{array}{c}\text { Unitary } \\
\text { ESPRIT } \\
\text { average } \\
\text { runtime (s) }\end{array}$ & $\begin{array}{c}\text { ESPRIT } \\
\text { average } \\
\text { runtime (s) }\end{array}$ & $\begin{array}{c}\text { Proposed } \\
\text { method average } \\
\text { runtime (s) }\end{array}$ \\
\hline$M=N=10$ & 0.01023 & 0.01081 & 0.00089 \\
$M=N=15$ & 0.04329 & 0.05503 & 0.00132 \\
$M=N=20$ & 0.18280 & 0.27440 & 0.00234 \\
$M=N=25$ & 0.59890 & 0.94800 & 0.00397 \\
$M=N=30$ & 1.60200 & 2.90600 & 0.00654 \\
\hline
\end{tabular}

\section{Conclusion}

In this paper, we have developed a low complexity unitary ESPRIT method for estimating angle in the bistatic MIMO radar. Compared with the existing unitary ESPRIT and ESPRIT algorithms which require $O\left(\left(M^{2} N^{2} L+M^{3} N^{3}\right) / 4\right)$ and $O\left(M^{2} N^{2} L+M^{3} N^{3}\right)$ flops, respectively, our approach only needs $O\left(2 M N L K+M N K^{2}\right)$ flops, thereby being much more computationally effective, especially for the case of a large MIMO radar array. Moreover, extensive simulation results demonstrate that the estimation precision of the presented scheme is much higher by comparison with the ESPRIT method and very similar to the unitary ESPRIT algorithm. In the future research, the presented technique can be extended to a different application such as estimating angle in the monostatic MIMO radar.

\section{Data Availability}

The relevant data used to support the findings of this study are within the manuscript.

\section{Conflicts of Interest}

The authors declare that there are no conflicts of interest in this work.

\section{Acknowledgments}

The authors wish to express their sincere thanks to the Natural Science Research Plan in Shaanxi Province of China (General Program, no.2020JM-574), the Ph.D Science Foundation of Polytechnic University (no.107020313), the National Natural Science Foundation of China (no.62001352), and the Technology Innovation Guidance Project of Shaanxi Province of China (no.2020CGXNG-012) for their support.

\section{References}

[1] A. Hassanien and S. A. Vorobyov, "Transmit energy focusing for DOA estimation in MIMO radar with colocated antennas," IEEE Transactions on Signal Processing, vol. 59, no. 6, pp. 2669-2682, 2011.

[2] Y. L. Yang, X. P. Mao, Y. G. Hou, and G. J. Jiang, "2-D DOA estimation via correlation matrix reconstruction for nested L-shaped array," Digital Signal Processing, vol. 98, pp. 1-11, 2020. 
[3] C. J. Hung, "Fast DOA estimation algorithm based on a combination of an orthogonal projection and noise pseudoeigenvector approach," ISRN Signal Processing, vol. 2011, Article ID 751670, 8 pages, 2011.

[4] H. D. Yan, J. Li, and G. S. Liao, "Multitarget identification and localization using bistatic MIMO radar systems," EURASIP Journal on Advances in Signal Processing, vol. 2008, Article ID 973932, 8 pages, 2008.

[5] M. L. Bencheikn, Y. D. Wang, and H. Y. He, "Polynomial root finding technique for joint DOD and DOD estimation in bistatic MIMO radar," Signal Processing, vol. 90, pp. 27232730, 2010.

[6] D. F. Chen, B. X. Chen, and G. D. Qian, "Angle estimation using ESPRIT in MIMO radar," Electronics Letters, vol. 44, no. 12 , pp. $770-771,2008$

[7] M. L. Bencheikh and Y. Wang, "Joint DOD-DOA estimation using combined ESPRIT-MUSIC approach in MIMO radar," Electronics Letters, vol. 46, no. 15, pp. 1081-1083, 2010.

[8] G. Zheng, B. Chen, and M. Yang, "Unitary ESPRIT algorithm for bistatic MIMO radar," Electronics Letters, vol. 48, no. 3, pp. 179-181, 2012.

[9] B. Tang, J. Tang, Y. Zhang, and Z. D. Zhang, "Maximum likelihood estimation of DOD and DOA for bistatic MIMO radar," Signal Processing, vol. 93, pp. 1394-1357, 2013.

[10] W.-G. Tang, H. Jiang, and S.-X. Pang, "Grid-free DOD and DOA estimation for MIMO radar via duality-based 2D atomic norm minimization," IEEE Access, vol. 7, pp. 60827-60836, 2019.

[11] X. Zhang and D. Xu, "Angle estimation in bistatic MIMO radar using improved reduced dimension Capon algorithm," Journal of Systems Engineering and Electronics, vol. 24, no. 1, pp. 84-89, 2013.

[12] J. Shi, G. Hu, X. Zhang, and F. Sun, "Sparsity-based DOA estimation of coherent and uncorrelated targets with flexible MIMO radar," IEEE Transactions on Vehicular Technology, vol. 68 , no. 6 , pp. 5835-5848, 2019.

[13] H. Zhang, W. Liu, Z. Zhang, W. Lu, and J. Xie, "Joint target assignment and power allocation in multiple distributed MIMO radar networks," IEEE Systems Journal, vol. 15, no. 1, pp. 694-704, 2021.

[14] X. Zhang, C. Chen, and J. Li, "Angle estimation using quaternion-ESPRIT in bistatic MIMO-radar," Wireless Personal Communications, vol. 69, no. 2, pp. 551-560, 2013.

[15] E. Fishler, A. Haimovich, R. Blum et al., "An idea whose time has come," in Proceedings of the 2004 International Radar Conference, pp. 71-78, Delft, Netherlands, April 2004.

[16] J. Li and P. Stoica, "MIMO radar with colocated antennas," IEEE Signal Processing Magazine, vol. 24, no. 5, pp. 106-114, 2007.

[17] B. Liao, "Fast angle estimation for MIMO radar with nonorthogonal waveforms," IEEE Transactions on Aerospace and Electronic Systems, vol. 54, no. 4, pp. 2091-2096, 2018.

[18] H. Ali, S. Ahmed, M. S. Sharawi, M. S. Alouini, and T. Y. AlNaffouri, "Reduced complexity DOA and DOD estimation for a single moving target in bistatic MIMO radar," Signal Processing, vol. 166, pp. 1-11, 2020.

[19] Y. Cheng, R. Yu, H. Gu, and W. Su, "Multi-SVD based subspace estimation to improve angle estimation accuracy in bistatic MIMO radar," Signal Processing, vol. 93, no. 7, pp. 2003-2009, 2013.

[20] X. Zhang and D. Xu, "Angle estimation in MIMO radar using reduced-dimension Capon," Electronics Letters, vol. 46, no. 12, pp. 860-861, 2010.
[21] X. Zhang, L. Xu, L. Xu, and D. Xu, "Direction of departure (DOD) and direction of arrival (DOA) estimation in MIMO radar with reduced-dimension MUSIC," IEEE Communications Letters, vol. 14, no. 12, pp. 1161-1163, 2010.

[22] M. Haardt and J. A. Nossek, "Unitary ESPRIT: how to obtain increased estimation accuracy with a reduced computational burden," IEEE Transactions on Signal Processing, vol. 43, no. 5, pp. 1232-1242, 1995.

[23] B. Xu and Y. Zhao, "Transmit beamspace based unitary parallel factor method for DOD and DOA estimation in bistatic MIMO radar,” IEEE Access, vol. 6, pp. 65573-65581, 2018.

[24] Y. D. Guo, Y. S. Zhang, and N. N. Tong, "Beamspace ESPRIT algorithm for bistatic MIMO radar," Electronics Letters, vol. 47, no. 15, pp. 876-878, 2011.

[25] A. Nicholas and J. W. Patrick, "Estimating principal components of large covariance matrices using the NystrÖm method," in Proceedings of the IEEE International Conference on Acoustics, Speech, and Signal Processing (ICASSP), pp. 3784-3787, Prague, Czech Republic, May 2011.

[26] P. Drineas and W. M. Micheal, "On the NystrÖm method for approximating a gram matrix for improved kernel-based learning," Journal of Machine Learning Research, vol. 6, pp. 2153-2175, 2005.

[27] C. K. I. Williams and M. Seeger, "Using the NystrÖm method to speed up kernel machines," Advances in Neural Information Processing Systems 2000, MIT Press, Cambridge, MA, USA, 2001.

[28] C. Fowlkes, S. Belongie, F. Chung, and J. Malik, "Spectral grouping using the nystrom method," IEEE Transactions on Pattern Analysis and Machine Intelligence, vol. 26, no. 2, pp. 214-225, 2004.

[29] C. Qian, L. Huang, and H. C. So, "Computationally efficient ESPRIT algorithm for direction-of-arrival estimation based on Nyström method," Signal Processing, vol. 94, pp. 74-80, 2014.

[30] J. P. Shi, F. Q. Wen, and T. P. Liu, "Nested MIMO radar: coarrays, tensor modeling and angle estimation," IEEE Transactions on Aerospace and Electronic Systems, vol. 57, no. 1, pp. 574-585, 2021. 